九州大学学術情報リポジトリ

Kyushu University Institutional Repository

\title{
A simple table lookup method for PET/CT partial volume correction using a point-spread function in diagnosing lymph node metastasis
}

Sakaguchi, Yuichi

Department of Health Sciences, Graduate School of Medical Sciences, Kyushu University

Mizoguchi, Noriko

Department of Medical Technology, Kyushu University Hospital

Mitsumoto, Tatsuya

Department of Health Sciences, Graduate School of Medical Sciences, Kyushu University

Mitsumoto, Katsuhiko

Department of Health Sciences, Graduate School of Medical Sciences, Kyushu University 他

http://hdl. handle. net/2324/26103

出版情報：Annals of Nuclear Medicine. 24 (8)，pp.585-591，2010-10-01. Springer Japan バージョン:

権利関係: (C) The Japanese Society of Nuclear Medicine 2010 


\section{ORIGINAL ARTICLE}

$<$ Original article $>$

A simple table lookup method for PET/CT partial volume correction using a

point-spread function in diagnosing lymph node metastasis

$<$ Short title $>$ PV correction by PSF for PET/CT

Yuichi Sakaguchi, $\mathrm{MS}^{1)}$, Noriko Mizoguchi, $\mathrm{BS}^{2)}$, Tatsuya Mitsumoto, $\mathrm{MS}^{1)}$, Katsuhiko Mitsumoto, $\mathrm{BS}^{1)}$, Kazuhiko Himuro ${ }^{2)}$, Nobuyoshi Ohya, $\mathrm{PhD}^{2)}$, Koichiro Kaneko, MD, $\mathrm{PhD}^{3)}$, Shingo Baba, $\mathrm{MD}, \mathrm{PhD}^{3)}$, Koichiro Abe, $\mathrm{MD}, \mathrm{PhD}^{3)}$, Yoshihiko Onizuka, $\mathrm{PhD}^{1)}$, Masayuki Sasaki, $\mathrm{MD}, \mathrm{PhD}^{1)}$

1) Division of Radiological Science, Department of Health Sciences, Graduate School of Medical Sciences, Kyushu University

2) Department of Medical Technology, Kyushu University Hospital

3) Department of Radiology, Kyushu University Hospital

3-1-1 Maidashi, Higashi-ku, Fukuoka 812-8582, Japan 
Phone: +81-92-642-6746, Fax: +81-92-642-6674

Corresponding author:

Masayuki Sasaki, MD, PhD

E-mail: msasaki@shs.kyushu-u.ac.jp 


\section{ABSTRACT}

Objective: We evaluated the partial volume effect in PET/CT images and developed a simple correction method to address this problem.

Methods: Six spheres and the background in the phantom were filled with

F-18 and we thus obtained 4 different sphere-to-background (SB) ratios.

Thirty-nine cervical lymph nodes in 7 patients with papillary thyroid carcinoma (15 malignant and 24 benign) were also examined as a preliminary clinical study. First, we developed recovery coefficient (RC) curves normalized to the maximum counts of the $37-\mathrm{mm}$ sphere. Next, we developed a correction table to determine the true SB ratio using three parameters, including the maximum counts of both the sphere and background and the lesion diameter, by modifying the approximation formula of the RC curves including the point-spread function correction. The full width at half maximum (FWHM) in this formula is estimated with the function of the SB ratio.

Results: In the phantom study, a size-dependent underestimation of the radioactivity was observed. The degree of decline of $\mathrm{RC}$ was influenced by the SB ratio. In preliminary clinical examination, the difference in the 
$\mathrm{SUV}_{\max }$ between malignant and benign LNs thus became more prominent after the correction. The PV correction slightly improved the diagnostic accuracy from $95 \%$ to $100 \%$.

Conclusions: We developed a simple table lookup correction method for the partial volume effect of PET/CT. This new method is considered to be clinically useful for the diagnosis of cervical LN metastasis. Further examination with a greater number of subjects is required to corroborate its clinical usefulness.

\section{KEY WORDS:}

PET/CT, lymph node metastasis, point-spread function, partial volume effect, recovery coefficient 


\section{INTRODUCTION}

[F-18]-2-deoxy-2-fluoro-D-glucose (FDG) - positron emission tomography (PET) is widely used for diagnosis, staging, restaging and monitoring therapy of many malignant tumors ${ }^{1)}$. Accurate diagnosis of lymph node metastasis is quite important because the $\mathrm{N}$-staging plays a key role in determining the therapeutic strategy of most malignancies. The diagnostic ability of FDG-PET for lymph node metastasis is generally considered to be higher than that of conventional morphological imaging modalities $^{2)}$. In lung cancer, we found a sensitivity of $76 \%$ and a specificity of $98 \%^{2)}$. A meta-analysis showed a median sensitivity of $85 \%$ and median specificity of $90 \%$ for FDG-PET ${ }^{3)}$. These are $10 \%$ to $20 \%$ higher than those for CT. In head and neck cancer, FDG-PET sensitivity was $79 \%$ and specificity $86 \%$ for detecting cervical lymph node metastasis ${ }^{4)}$. These are also higher than those of CT. However, the poor spatial resolution in PET systems results in limitations for the detection of small malignant lesions especially in metastatic lymph nodes. Gould et al. reported a lower sensitivity in small lymph nodes in comparison with that in large lymph nodes ${ }^{3)}$. The standardized uptake values (SUV) of small lesions are 
usually underestimated due to the partial volume (PV) effects, thus resulting in false negative findings.

Although several methods have been proposed to correct the PV effects of PET images, such as the geometric transfer matrix (GTM) method $^{5,6)}$ and the fitting method ${ }^{7)}$, they are not clinically applicable because they require complex processes. A recovery coefficient (RC) method $^{8-11)}$ is a relatively convenient method using a simple conversion formula; however, these correction methods require the diameter of the lesion to calculate the true radioactivity. The precise diameters of lesions cannot be obtained from PET images making other morphological diagnostic examinations, such as CT, MRI or US, necessary. Although software fusion of separately performed PET and CT images can provide the same information, it is cumbersome and is not routinely applicable in all institutions. Differences in patient status or patient position between the two examinations may cause mislocation of small lesions. Recently, advances in PET/CT technology have resolved these problems; now, morphological and metabolic information can be obtained simultaneously. The radioactivity of the lesion can be determined from PET images and the 
size of the lesion from CT images. Furthermore, previously-reported RC methods have been developed based on the limited lesion-to-background ratio, which was considered to influence the spatial resolution of PET images.

The purpose of this study is to develop a simple correction method for the PV effect in a PET/CT system to improve the diagnosis of lymph node metastasis. For this purpose, we investigated the PV effect in different lesion-to-background ratios and employed the point-spread function based on simultaneously obtained morphological and metabolic information.

\section{MATERIALS AND METHOD}

\section{Phantom Study}

The National Electrical Manufacturers Association (NEMA) 2001 International Electrotechnical Commission (IEC) Body Phantom consisting of a quasi-cylindrical cavity $(280 \times 210 \times 180 \mathrm{~mm})$ and six spheres (Model ECT/IEC-BODY/P) was used for this study (Data Spectrum Corp, Hillsborough, NC). These spheres were 37, 28, 22, 17, 13 and 10mm in 
inner diameter, and their wall thickness was $1 \mathrm{~mm}$. All of the spheres were filled with ${ }^{18} \mathrm{~F}$ solution of $34.2 \mathrm{kBq} / \mathrm{mL}$. The phantom background was filled with ${ }^{18} \mathrm{~F}$ solution of different concentrations to yield sphere-to-background (SB) ratios of around 10:1, 5:1, 2:1 and 1.3:1. The true SB ratios were 7.59:1, 4.02:1, 2.17:1 and 1.25:1. The ratios are expressed as the radioactivity of the $37-\mathrm{mm}$ sphere to the background.

\section{Preliminary clinical study}

The subjects consisted of 7 patients with papillary thyroid carcinoma who underwent total thyroidectomy. All patients were referred for radioiodine therapy because of clinically suspected lymph node metastasis. PET/CT was performed before therapy. On PET/CT images, FDG accumulation in cervical lymph nodes was classified as positive, equivocal or negative by visual evaluation by 2 nuclear medicine physicians. This resulted in nine positive, seventeen equivocal and 13 negative lymph nodes being retrospectively enrolled in this study. Lesions consisted of 39 cervical lymph nodes and were finally diagnosed as 15 metastatic ones $(9$ visually positive and 6 visually equivocal) and 24 benign ones (11 visually 
equivocal and 13 visually negative). Of 15 metastatic lymph nodes, 13 were pathologically diagnosed and 2 were clinically diagnosed because of the interval increase in size during 12-month follow-up. All of the 24 benign lymph nodes were clinically diagnosed because they showed negative serum thyroglobulin level, negative radioiodine accumulation and no interval increase in size for at least 26 months follow-up. The short-axis diameter in the $\mathrm{CT}$ images ranged from 2.04 to $11.6 \mathrm{~mm}$ (mean $6.89 \pm 2.20 \mathrm{~mm})$. That of metastatic lymph nodes $(6.26 \pm 1.61 \mathrm{~mm}$, range from 4.3 to $9.3 \mathrm{~mm})$ did not differ from that of benign ones $(7.28 \pm 2.45 \mathrm{~mm}$, 3.4 to $11.6 \mathrm{~mm}$ ). This study was approved by the review board of the Ethics Committee of our institution. This study is retrospective in nature, and its results did not influence further therapeutic decision-making.

\section{Data Acquisition}

Data acquisition was performed with a Discovery ST Elite PET/CT system (GE Healthcare, Milwaukee, WI, USA). The 16-slice CT scanning parameters were $120 \mathrm{kv}, 50 \mathrm{~mA}, 512 \times 512$ matrix, slice thickness of $5 \mathrm{~mm}$, transaxial field of view of $500 \mathrm{~mm}$ and $0.5 \mathrm{sec} /$ rotation of gantry rotation. 
The PET scanner is comprised of 24 ring detectors consisting of 560 BGO crystal $(4.7 \times 6.3 \times 30 \mathrm{~mm})$. The transverse and axial field of views are $700 \mathrm{~mm}$ and $157 \mathrm{~mm}$, respectively. PET emission data were acquired in the 3D mode with $128 \times 128$ matrices $(5.47 \times 5.47 \times 3.27 \mathrm{~mm})$. The acquisition times per bed position were three minutes in static mode. In the phantom study, thirty frames at ten-minute intervals were acquired in a bed position. In the clinical study, all patients fasted for at least 4 hours before injection of 180-370 MBq of ${ }^{18}$ F-FDG. The conventional whole-body PET/CT scan was acquired for each patient from the top of the skull to the middle of the thigh sixty minutes after intravenous administration. The whole-body PET/CT was performed in 7-8 bed positions with 9 overlapping slices. PET was reconstructed using a 3D ordered subsets expectation maximization (3D-OSEM) algorithm (VUE Point Plus, 2 iterations, 28 subsets, and a post-filter of 6-mm full width at half maximum (FWHM).

\section{Data Analysis}

\section{Phantom study}

The counts in each sphere were determined by the following 
process to minimize the statistical variance of the count. A circular region of interest (ROI) with a diameter corresponding to the known physical diameter of the sphere was placed on each sphere image. First, the time activity curves (TACs) of each sphere were generated using dynamic acquisition data and were fitted by exponential functions. Next, a frame with a concentration of $18 \mathrm{kBq} / \mathrm{mL}$ in a $37-\mathrm{mm}$ sphere on the approximated TAC was selected to use for analysis. The maximum counts of each sphere and background were measured in the axial central slice.

The $\mathrm{RC}$ of each sphere was expressed as ratios of the maximum radioactivity of each sphere to that of the 37-mm sphere for each SB ratio and then the RC curves were generated. RC curves were considered to be an integral of the Gaussian weighting function because the point-spread function (PSF) is essentially Gaussian ${ }^{12)}$. According to the report of Kessler et al. ${ }^{13)}$, approximated RC curves were generated using an integral of the Gaussian function as a function of the radius of the hot sphere and the FWHM:

$$
F_{g s}(R / \sigma, 0)=\operatorname{erf}(R / \sqrt{2} \sigma)-\sqrt{2 / \pi} *(R / \sigma) * \mathrm{e}^{-\left[(\mathrm{R})^{2} / 2 \sigma^{2}\right]}, \quad \text { Eq. } 1
$$

where $R$ represents the radius of the hot sphere and $\sigma$ the standard deviation 
of the Gaussian function. The relationship between $\sigma$ and FWHM is straightforward:

FWHM $/(2 \sigma)=(2 \times \ln 2)^{0.5} \fallingdotseq 1.18$

$\sigma=\mathrm{FWHM} / 2.35$

Furthermore, the FWHM of PET system is also thought to be influenced by the SB ratio. We developed a function to obtain the FWHM in response to the SB ratio. First, the FWHM of each SB ratio was calculated by fitting the RC curve of each SB ratio to equation 1 . Next, the relationship between the SB ratio and FWHM was approximated by the following equation, similar to the hyperbolic function (Figure 1). When the background was filled with no radioactivity, the FWHM was close to 0.7 $\mathrm{cm}$. Therefore, the function to estimate the FWHM was as follows:

$$
\mathrm{FWHM}=0.7+1 /\left(0.35 \times(\mathrm{SB} \text { ratio }+0.4)^{1.95}\right) \quad \text { Eq. } 2
$$

A lookup table to find the true SB ratio was created based on equations 1 and 2 (Figure 2). The length of the table shows the radius of the lesion, and the side of the table shows the true SB ratio. The numbers in the chart represent the measured SB ratios (yielded in the PET images). The procedure for obtaining the corrected counts of objects is as follows. First, 
the radius of the lesions (1), maximum counts of the objects (2) and the background counts (3) must be obtained from PET-CT images. Second, the measured SB ratio (4) should be calculated by the maximum counts of the object over the background counts ((2) / (3)). Third, the corresponding radius (1) in the left end-column of the lookup table is found. Fourth, the corresponding measured SB ratio (4) in the row of the corresponding radius is found. Fifth, the true SB ratio (5) can be found in the uppermost row of the corresponding measured SB ratio. Finally, the true counts can be calculated as the product of the measured background counts and the true SB ratio ( (3)x(5)).

\section{Preliminary clinical study}

The clinical data analysis was performed in 39 cervical lymph nodes on PET-CT images using Xeleris (GE Healthcare, Milwaukee, WI, USA). After determination of a cervical lymph node on CT images, its short-axis diameter was measured. A circular region-of-interest (ROI) for the lymph node was placed to surround it on CT images, and the highest pixel value on the corresponding PET image was determined as the 
SUVmax of the lymph node. A circular ROI of the same size was placed on the cervical muscle on the contralateral side of the intended lymph node for background. The average SUV in the ROI was determined and used as the background activity.

\section{Statistical analysis}

The difference in the SUVmax between malignant and benign lymph nodes was analyzed by $t$ test. The relationship between the size and SUVmax of lymph nodes was analyzed with Pearson's correlation coefficient test. Comparison of diagnostic ability was analyzed by McNemar's chi-square test. $\mathrm{P}$ values less than 0.05 were considered to indicate statistical significance.

\section{RESULTS}

\section{Phantom study}

On the PET images, all spheres could be visually recognized in the actual SB ratio of 7.59, 4.02 and 2.17 (Figure 3). However, the smaller the spheres, the more faint the images. The spheres with a diameter of $17 \mathrm{~mm}$ 
or smaller could not be detected with an SB ratio of 1.25. The RCs gradually decreased in a smaller diameter due to the PV effect. The RC curve, background activities and approximated RC curves yielded by equation 1 of each SB ratio are shown in Figure 4. The patterns of the RC curves were different among the SB ratios. The slopes of the $\mathrm{RC}$ curves were gentle when the SB ratio was low. The RC curves approximated by equation 1 showed excellent approximation in every SB ratio. The RCs after the PV correction showed an improvement of the size-dependent underestimation.

\section{Preliminary clinical study}

The relationship between the size and SUVmax of the lymph nodes before and after PV correction are shown in Figure 5. Before PV correction, the SUVmax of the malignant lymph nodes $(6.35 \pm 2.09)$ was higher than that of the benign lymph nodes $(2.04 \pm 1.16)$. The difference was statistically significant $\left(\mathrm{p}=6.09 \times 10^{-3}\right)$. After PV correction, the difference between the SUVmax of the malignant and benign lymph nodes became more prominent $\left(20.49 \pm 5.25\right.$ vs. $\left.4.88 \pm 2.41, \mathrm{p}=4.92 \times 10^{-4}\right)$. 
The positive correlation between the $\mathrm{SUV}_{\max }$ and the diameter of both malignant and benign lymph nodes $\left(\mathrm{p}=4.85 \times 10^{-4}, 3.89 \times 10^{-5}\right)$ disappeared after the correction. The SB ratio of all lymph nodes was $1.61 \pm 0.57$ (range from 0.86 to 3.54 ) before PV correction and $4.83 \pm 2.68$ (1.00 to 11.89) after PV correction. The SB ratios were less than 4.0 in all 39 lymph nodes before correction (100\%) and 20 after correction (51\%). The diagnostic accuracy was $95 \%$ (sensitivity $100 \%$, specificity $92 \%$, positive predictive value $88 \%$, negative predictive value $100 \%$ by cutoff SUV 4.00) before PV correction and was 100\% (100\%, 100\%, 100\%, $100 \%$ by cutoff SUV 10) after PV correction, respectively. The difference was not significant.

\section{DISCUSSION}

This study evaluated the PV effect of a PET/CT system and developed a simple correction method using simultaneously obtained morphological and metabolic information in the cervical lymph nodes in patients with papillary thyroid cancer. Recent developments in PET/CT technology have enabled us to obtain both the radioactivity and size of the 
object simultaneously. Underestimation of the radioactivity due to the PV effect was observed in small objects; the smaller the size, the larger the degree of decline. The degree of decline is dependent on the SB ratio; thus, we created an equation to estimate the FWHM with the function of the SB ratio. Our correction method using PSF requires 3 kinds of clinical data measured by PET/CT: the radius of the lesion, the maximum radioactivity of the lesion and the background radioactivity.

In the PET system, the radioactivity of objects with a diameter of FWHM $\times 3$ or smaller is generally underestimated ${ }^{14)}$. However, this phenomenon is observed only in low background activity. Kessler et al. proposed an equation using PSF for the theoretical explanation of the PV effect and suggested that it would be used only in a case without any radioactive background ${ }^{13)}$. We observed that the peak value of the PSF decreased and its shape became broad in a radioactive background. We also observed that the FWHM increased when the radioactivity of the background increased. Srinivas et al. evaluated the RCs in 3 different SB ratios and reported that they were only weakly influenced by the SB ratio of $8: 1,6: 1$ and $4: 1^{11)}$. Although no grossly different pattern was observed in 
the RCs with the SB ratios of 8:1 and 4:1 in our study, quite different patterns were observed in the RC curves with the SB ratio of $2.17: 1$ and 1.25:1. The SUVs of lesions with such low SB ratios are supposed to be less than 2.5 , and such lesions must provide the most troubling aspect in the clinical interpretation especially in small lesions. In this study, the SB ratio of subjects was $1.61 \pm 0.57$ and was less than 4.0 in all 39 lymph nodes $(100 \%)$ before correction. We thus considered that the low SB ratio must be important for PV correction and built a function to calculate FWHM by the SB ratio into the correction method.

Previous studies proposed several methods for PV correction. The geometric transfer matrix (GTM) method is considered to be relatively accurate, but it requires high-resolution MRI images, and its clinical application is very cumbersome ${ }^{5,6}$. The fitting method requires many parameters for calculation ${ }^{7}$. Our correction method is considered to be a kind of RC method, which is known to be relatively convenient. In an equation to correct the PV effect, the $\mathrm{RC}$ at the object size was determined from the RC curve obtained by their experiments. Although most conversion formulas use a directly proportional relationship ${ }^{8-11)}$, the RC 
curve is normally sigmoid-shaped and is not a straight line. The process for the PV correction in their methods is simple, though they are considered to emphasize the error in small lesions ${ }^{8)}$. On the other hand, we approximated the RC curves by using PSF to minimize the variance of radioactivity measurement in our method. Furthermore, we established an equation to determine the FWHM based on a different SB ratio. Although we could not establish a conversion function to calculate the true radioactivity directly, we made a lookup table to find the true SB ratio.

In this study, we targeted cervical lymph nodes as the focus of our study. The morphological diagnostic criterion for cervical lymph node metastasis is generally a short-axis diameter of $10 \mathrm{~mm}$ or larger ${ }^{15)}$. Although the diagnostic ability of FDG-PET is superior to that of CT, equivocal findings are often observed in lymph nodes with a diameter around $10 \mathrm{~mm}$. Thus, PV correction for cervical lymph nodes is expected to improve the diagnostic ability of PET/CT. Our correction method includes a PSF, which is a 3-dimensional integral of a Gaussian function. Strictly speaking, our correction method should thus be used for refined spherical-shaped lesions. Clinically, most malignant tumors have an 
irregular shape and are not considered to be appropriate for our method. On the contrary, most metastatic lymph nodes, especially small nodes, seem to be either spheroid-shaped or at least oval-shaped; thus, we considered our method to be acceptable for the differential diagnosis of lymph node metastasis. Another important reason is that cervical lymph nodes do not move during data acquisition. Lesions in the trunk can move due to respiration and heart beat, resulting in image blurring and smearing. The reason radioactivity is underestimated in such moving lesions is complex and cannot be corrected by a method based only on size-dependent theory.

The distribution of radioactivity in the phantom is homogeneous in this study. In clinical examination, neoplastic tissue consists of histologically different components resulting in heterogeneous FDG accumulation in the tumor. If a lesion has a non-uniform distribution of radioactivity, this correction method may not be applicable because the RC may be different from that of the phantom study. The second problem is that this correction method lacks versatility. The RC curves are specific for the PET device, the data acquisition protocol, the size of the matrix, the reconstruction methods and the parameters for reconstruction. The lookup 
table for PV correction must be established not only in each device but also in each examination protocol. The 3rd problem is the limited number of subjects in clinical study, although it was a preliminary examination. Further examination with a greater number of subjects with various SUV is required to corroborate the usefulness of this method.

\section{CONCLUSION}

We established a simple table lookup correction method for PET/CT PV correction using PSF including an equation for the estimation and an FWHM based on the SB ratio. This new method is considered to be useful for the diagnosis of cervical LN metastasis in a limited number of patients with thyroid cancer. Further examination with a greater number of subjects is required to corroborate the clinical usefulness of this method. 


\section{ACKNOWLEDGMENT}

The authors thank the radiological technologists in the Division of Nuclear

Medicine of Kyushu University Hospital and Mr. Hirofumi Kawakami for their valuable technical assistance. 


\section{REFERRENCES}

1 Poeppel TD, Krause BJ, Heusner TA, Boy C, Bockisch A, Antoch G. PET/CT for the staging and follow-up of patients with malignancies. Eur J Radiol. 2009; 70(3): 382-92.

2 Sasaki M, Ichiya Y, Kuwabara Y, Akashi Y, Yoshida T, Fukumura T, et al. The usefulness of FDG positron emission tomography for the detection of mediastinal lymph node metastases in patients with non-small cell lung cancer: a comparative study with X-ray computed tomography. Eur J Nucl Med. 1996; 23: 741-7.

3 Gould MK, Kuschner WG, Rydzak CE, Maclean CC, Demas AN, Shigemitsu H, et al. Test performance of positron emission tomography and computed tomography for mediastinal staging in patients with non-small-cell lung cancer: a meta-analysis. Ann Intern Med 2003; 139(11): 879-92.

4 Kyzas PA, Evangelou E, Denaxa-Kyza D, Ioannidis JP. 18F-fluorodeoxyglucose positron emission tomography to evaluate cervical node metastases in patients with head and neck squamous cell carcinoma: a meta-analysis. J Natl Cancer Inst. 2008; 100(10): 
$712-20$.

5 Rousset OG, Ma Y, Evans AC. Correction for partial volume effects in PET: principle and validation. J Nucl Med 1998; 39: 904-11.

6 Rousset OG, Collins DL, Rahmim A, Wong DF. Design and implementation of an automated partial volume correction in PET: application to dopamine receptor quantification in the normal human striatum. J Nucl Med 2008; 49: 1097-106.

7 Chen CH, Muzic RF Jr, Nelson AD, Adler LP. Simultaneous recovery of size and radioactivity concentration of small spheroids with PET data. J Nucl Med 1999; 40: 118-30.

8 Adler LP, Crowe JP, al-Kaisi NK, Sunshine JL. Evaluation of breast masses and axillary lymph nodes with [F-18] 2-deoxy-2-fluoro-D-glucose PET. Radiology 1993; 187: 743-50.

9 Geworski L, Knoop BO, de Cabrejas ML, Knapp WH, Munz DL. Recovery correction for quantitation in emission tomography: a feasibility study. Eur J Nucl Med 2000; 27(2): 161-69.

10 Hickeson M, Yun M, Matthies A, Zhuang H, Adam LE, Lacorte L, Alavi A. Use of a corrected standardized uptake value based on the 
lesion size on CT permits accurate characterization of lung nodules on FDG-PET. Eur J Nucl Med Mol Imaging 2002; 29(12): 1639-47.

11 Srinivas SM, Dhurairaj T, Basu S, Bural G, Surti S, Alavi A. A recovery coefficient method for partial volume correction of PET images. Ann Nucl Med. 2009; 23(4): 341-8.

12 Hoffman EJ, Huang SC, Phelps ME. Quantitation in positron emission computed tomography: 1. Effect of object size. J Comput Assist Tomogr 1979; 3: 299-308.

13 Kessler RM, Ellis JR Jr, Eden M. Analysis of emission tomographic scan data: limitations imposed by resolution and background. $\mathrm{J}$ Comput Assist Tomogr. 1984; 8: 514-22.

14 Soret M, Bacharach SL, Buvat I. Partial-volume effect in PET tumor imaging. J Nucl Med. 2007; 48(6): 932-45.

15 Sigg MB, Steinert H, Grätz K, Hugenin P, Stoeckli S, Eyrich GK. Staging of head and neck tumors: $\left[{ }^{18} \mathrm{~F}\right]$ fluorodeoxyglucose positron emission tomography compared with physical examination and conventional imaging modalities. J Oral Maxillofac Surg 200; 61(9): 1022-9. 


\section{FIGURE LEGENDS}

Figure 1. Relationship between the measured SB ratio and FWHM. The relationship is approximated with a formula: $\mathrm{FWHM}=0.7+1 /$ $\left(0.35 \times(\mathrm{SB} \text { ratio }+0.4)^{1.95}\right)$.

Figure 2. The lookup table to find the true SB ratio. First, the radius measured by CT is found in the left end column (for example, $\mathrm{R}=1.50$ ). Second, the measured $\mathrm{SB}$ ratio is found in the row of the corresponding radius (measured $\mathrm{SB}=1.000280$ ). Third, the true SB ratio is found in the uppermost row of the corresponding measured $\mathrm{SB}$ ratio (true $\mathrm{SB}=1.4$ ).

Figure 3. PET images of IEC body phantom with 10-, 13-, 17-, 22-, 28and 37-mm-diameter hot spheres. The SB ratios were (A) 7.59:1, (B) 4.02:1, (C) 2.17:1 and (D) 1.25:1.

Figure 4. The measured and approximated RC curves for different SB 
ratio. The SB ratios were (A) 7.59:1, (B) 4.02:1, (C) 2.17:1 and (D) 1.25:1. Closed circle and dotted line, measured RC; closed triangle and dotted line, measured background; open circle and solid line, approximated RC curve.

Figure 5. Relationship between the short-axis diameter and the SUVmax of lymph nodes. (Closed square, malignant with visually positive; closed circle, malignant with visually equivocal; open circle, benign with visually equivocal; open triangle, benign with visually negative). A. Before PV correction, the SUVmax of the malignant lymph nodes $(6.35 \pm 2.09)$ was significantly higher than that of the benign lymph nodes (2.04 \pm 1.16) $\left(p=6.09 \times 10^{-3}\right)$. B. After PV correction, the difference between the SUVmax of the malignant and benign lymph nodes became more prominent $(20.49 \pm 5.25$ vs. $4.88 \pm 2.41, \mathrm{p}=4.92$ $\left.\times 10^{-4}\right)$. 
Figure 1

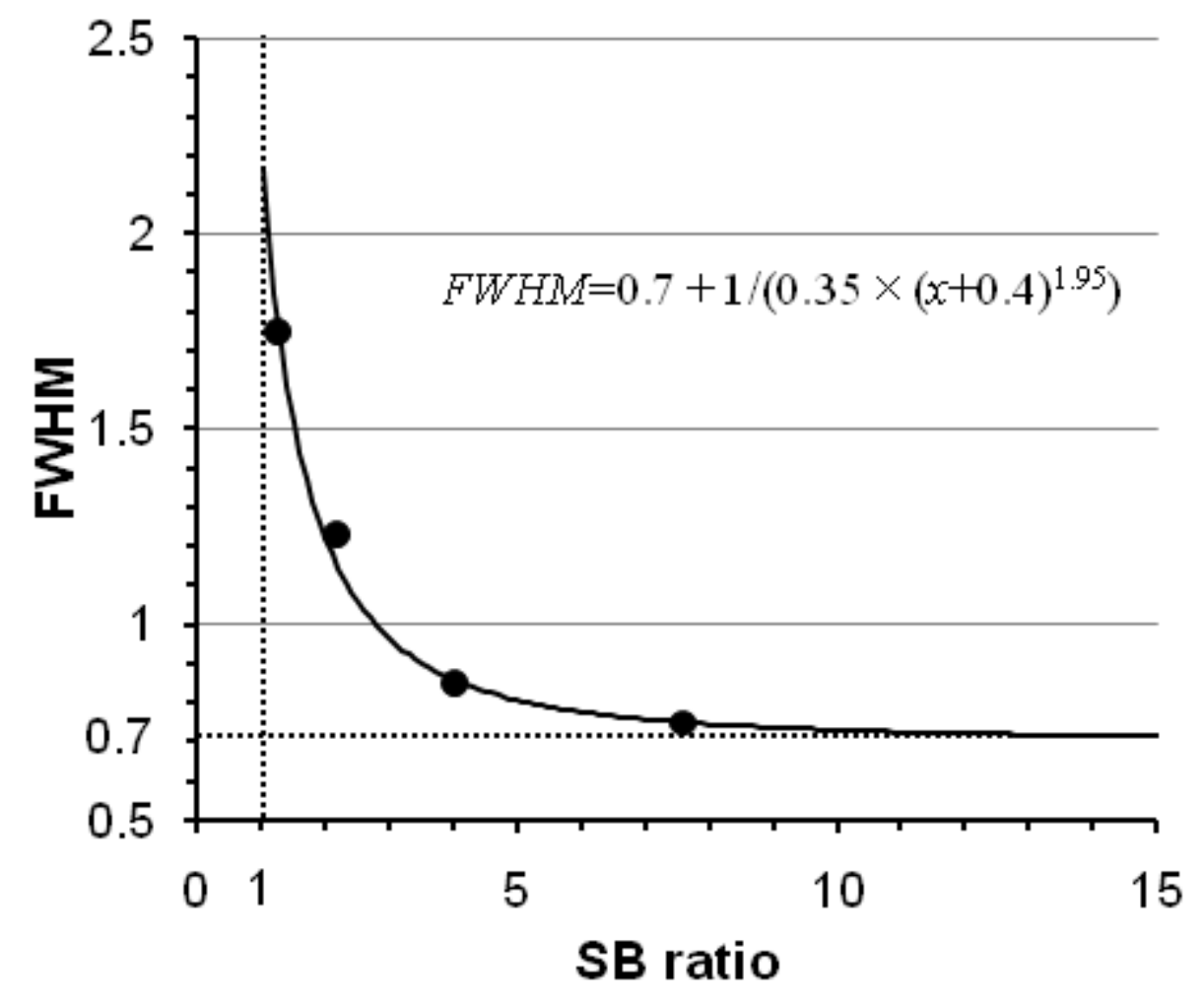


True SB raio

\begin{tabular}{|c|c|c|c|c|c|c|c|}
\hline $\mathrm{R}(\mathrm{mm})$ & 1.1 & 1.2 & 1.3 & 1.4 & 1.5 & 19.9 & 20.0 \\
\hline 0.00 & 1 & 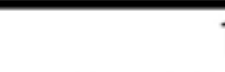 & 1 & 1 & 1 & 1.000000 & 1.000000 \\
\hline 1.00 & 1.000044 & 1.000032 & 1.000055 & $1.00 \not 083$ & 1.000116 & 1.180044 & 1.181054 \\
\hline 1.05 & 1.000051 & 1.000037 & 1.000064 & $1.00 p 096$ & 1.000134 & 1.207718 & 1.208883 \\
\hline 1.10 & 1.000058 & 1.000043 & 1.000073 & $1.00 p 111$ & 1.000154 & 1.237979 & 1.239314 \\
\hline 1.15 & 1.000067 & 1.000049 & 1.000084 & $1.00 p 126$ & 1.000176 & 1.270919 & 1.272438 \\
\hline 1.20 & 1.000076 & 1.000055 & 1.000095 & $1.00 p 143$ & 1.000200 & 1.306622 & 1.308342 \\
\hline 1.25 & 1.000085 & 1.000062 & 1.000108 & $1.00 p 162$ & 1.000226 & 1.345171 & 1.347106 \\
\hline 1.30 & 1.000096 & 1.000070 & 1.000121 & $1.00 p 182$ & 1.000254 & 1.386641 & 1.388808 \\
\hline 1.35 & 1.000107 & 1.000079 & 1.000135 & 1.00 p204 & 1.000284 & 1.431104 & 1.433521 \\
\hline 1.40 & 1.000120 & 1.000088 & 1.000151 & $1.00 \mathrm{~b} 228$ & 1.000317 & 1.478629 & 1.481312 \\
\hline 1.45 & 1.000133 & 1.000097 & 1.000168 & $1.00 \mathrm{p} 253$ & 1.000352 & 1.529277 & 1.532243 \\
\hline 1.50 & 1.000147 & 1.000100 & 1.0001007 & 1.000280 & 1.000389 & 1.583106 & 1.586373 \\
\hline 1.55 & 1.000162 & 1.000119 & 1.000205 & 1.000308 & 1.000429 & 1.640169 & 1.643755 \\
\hline 1.60 & 1.000178 & 1.000130 & 1.000225 & 1.000339 & 1.000472 & 1.700512 & 1.704435 \\
\hline 1.65 & 1.000195 & 1.000143 & 1.000247 & 1.000372 & 1.000518 & 1.764179 & 1.768458 \\
\hline 1.70 & 1.000213 & 1.000156 & 1.000270 & 1.000406 & 1.000566 & 1.831207 & 1.835860 \\
\hline 1.75 & 1.000233 & 1.000171 & 1.000294 & 1.000443 & 1.000617 & 1.901628 & 1.906674 \\
\hline 1.80 & 1.000253 & 1.000186 & 1.000320 & 1.000482 & 1.000671 & 1.975469 & 1.980927 \\
\hline 1.85 & 1.000274 & 1.000201 & 1.000347 & 1.000523 & 1.000728 & 2.052752 & 2.058641 \\
\hline 1.90 & 1.000297 & 1.000218 & 1.000376 & 1.000566 & 1.000788 & 2.133493 & 2.139832 \\
\hline 1.95 & 1.000321 & 1.000236 & 1.000406 & 1.000612 & 1.000852 & 2.217704 & 2.224513 \\
\hline 2.00 & 1.000346 & 1.000254 & 1.000438 & 1.000660 & 1.000919 & 2.305391 & 2.312688 \\
\hline 39.00 & 1.099991 & 1.197484 & 1.297688 & 1.398079 & 1.498473 & 19.900000 & 20.000000 \\
\hline 40.00 & 1.099995 & 1.198060 & 1.298266 & 1.398596 & 1.498912 & 19.900000 & 20.000000 \\
\hline
\end{tabular}




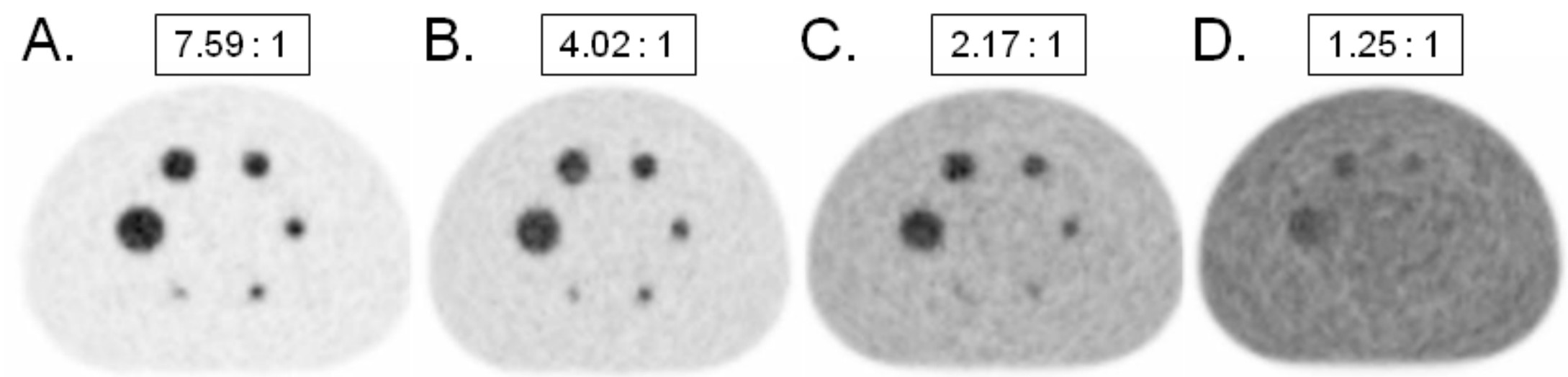




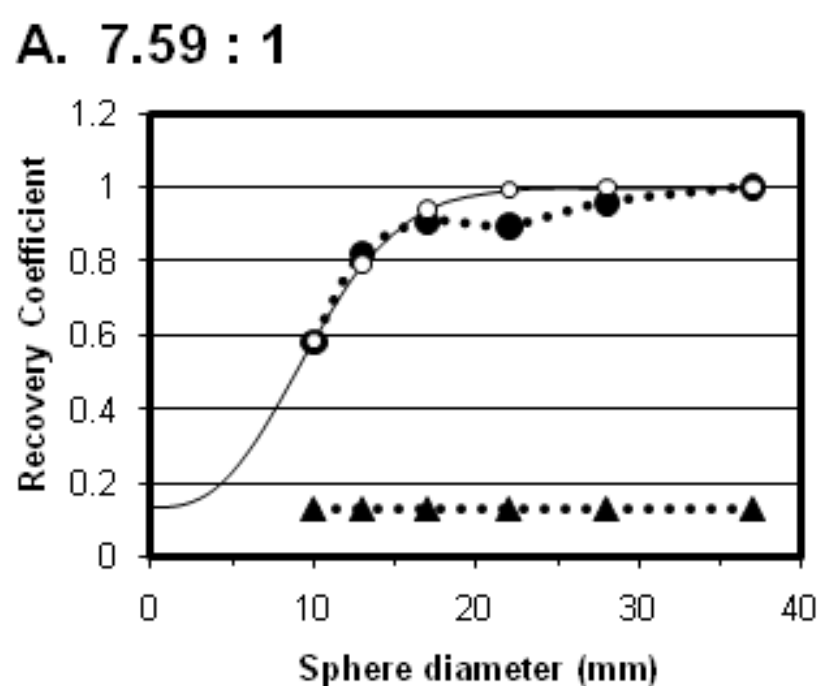

C. $2.17: 1$

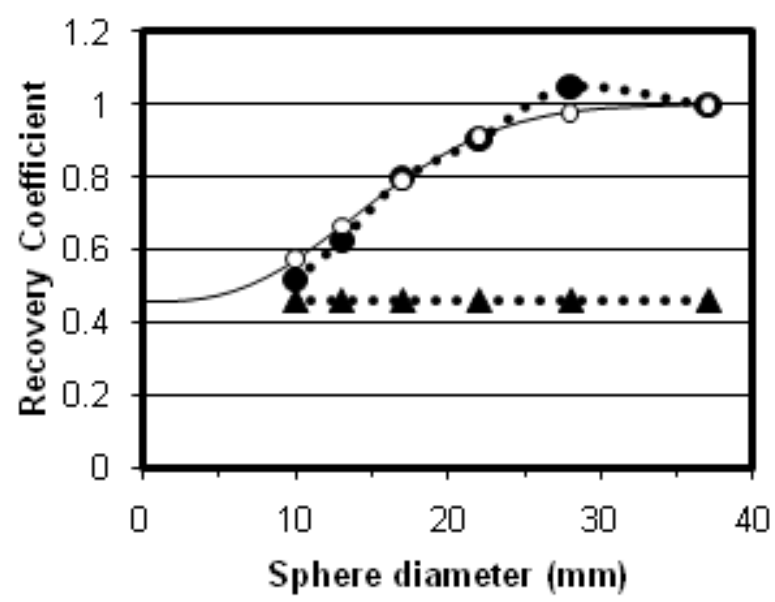

B. $4.02: 1$

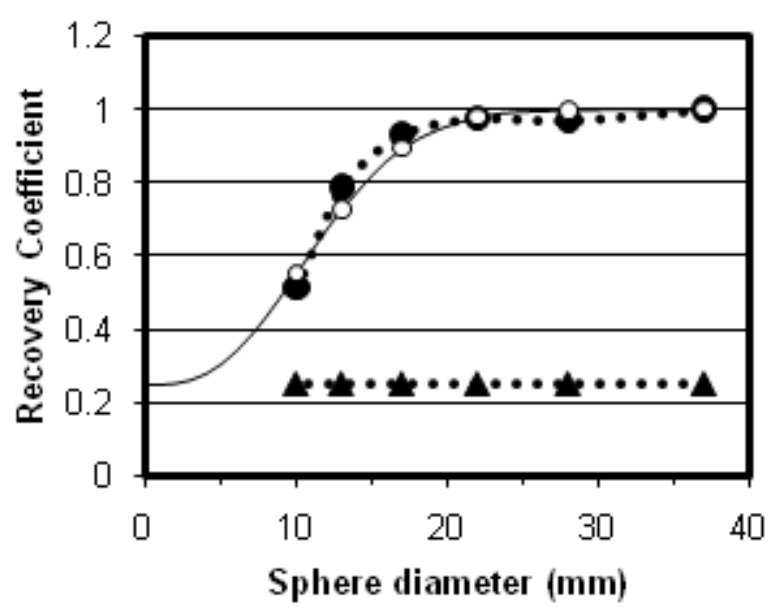

D. $1.24: 1$

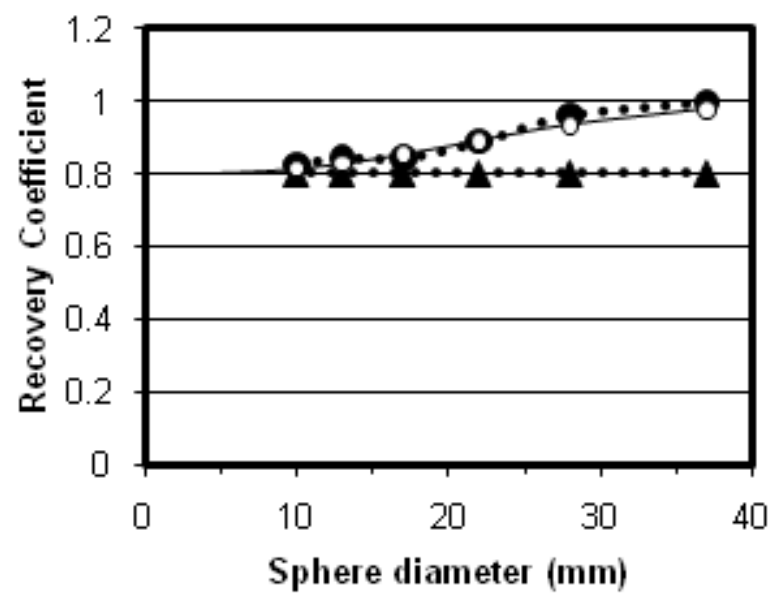




\section{Figure 5 re-revise}

A. Before PV correction

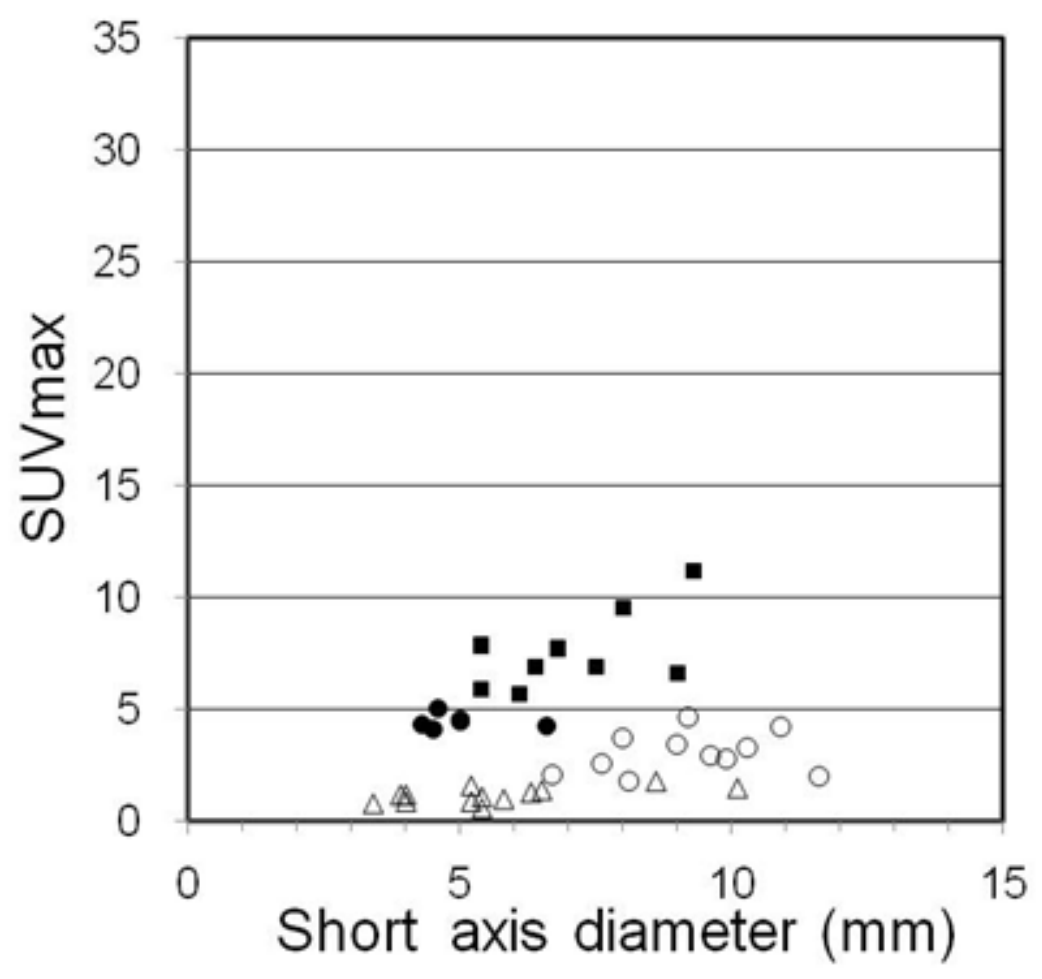

B. After PV correction

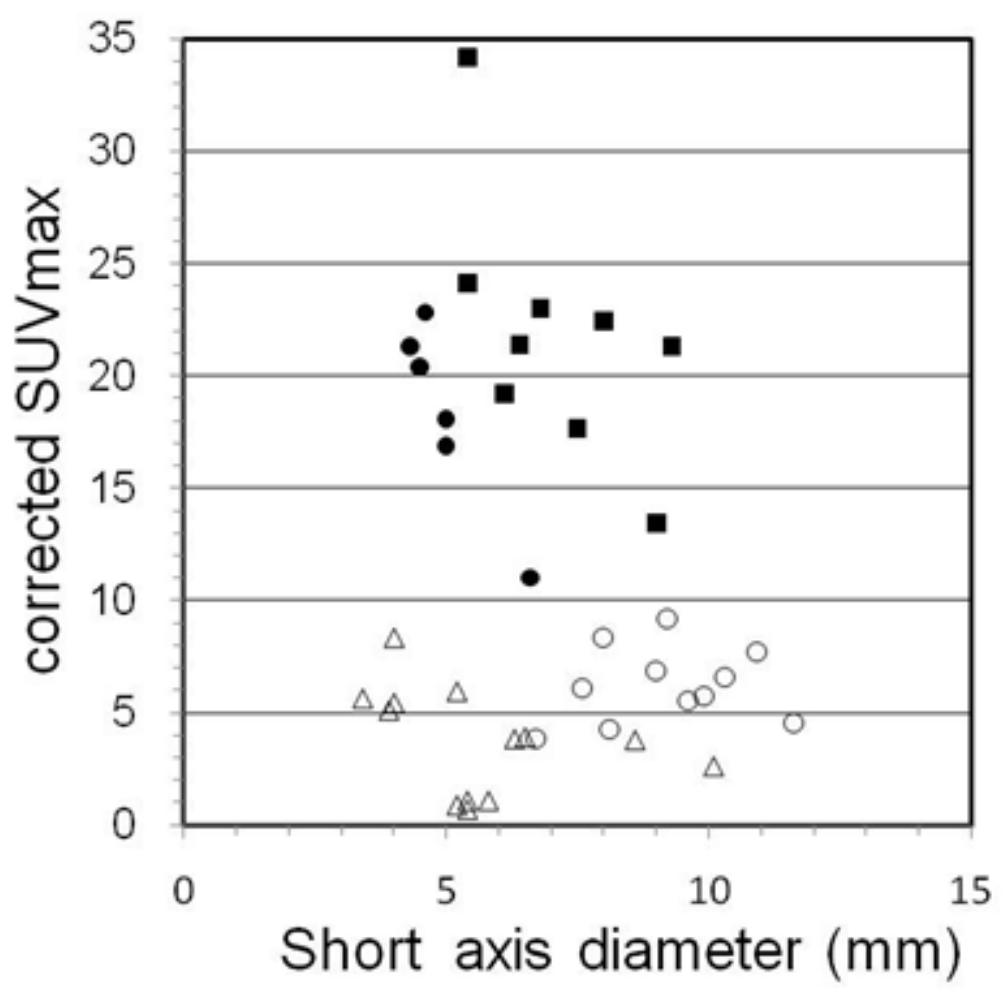

